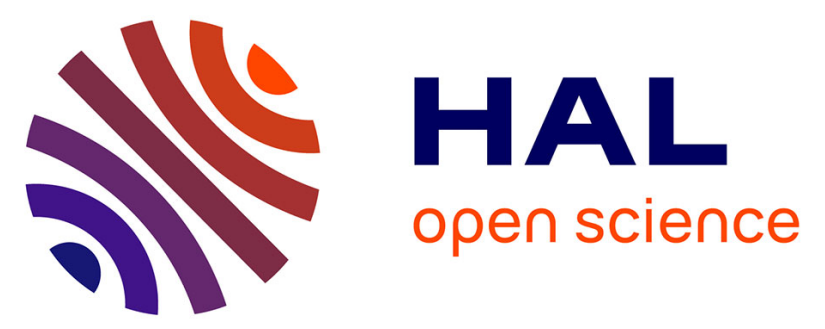

\title{
GOSPEL 3 prise en charge de la goutte par les médecins généralistes et rhumatologues libéraux en France au début du XXIe siècle. Comparaison aux recommandations EULAR 2006
}

J. Goossens, S. Lancrenon, S. Lanz, H.-K. Ea, C. Lambert, P. Guggenbuhl, A. Saraux, C. Delva, F. Lioté, S. Sahbane

\section{To cite this version:}

J. Goossens, S. Lancrenon, S. Lanz, H.-K. Ea, C. Lambert, et al.. GOSPEL 3 prise en charge de la goutte par les médecins généralistes et rhumatologues libéraux en France au début du XXIe siècle. Comparaison aux recommandations EULAR 2006. Revue du Rhumatisme, 2018, 85 (2), pp.165-172. 10.1016/j.rhum.2016.08.011 . hal-01777714

HAL Id: hal-01777714

https://hal-univ-rennes1.archives-ouvertes.fr/hal-01777714

Submitted on 7 Jun 2018

HAL is a multi-disciplinary open access archive for the deposit and dissemination of scientific research documents, whether they are published or not. The documents may come from teaching and research institutions in France or abroad, or from public or private research centers.
L'archive ouverte pluridisciplinaire HAL, est destinée au dépôt et à la diffusion de documents scientifiques de niveau recherche, publiés ou non, émanant des établissements d'enseignement et de recherche français ou étrangers, des laboratoires publics ou privés. 
GOSPEL 3 : Prise en charge de la goutte par les médecins généralistes et

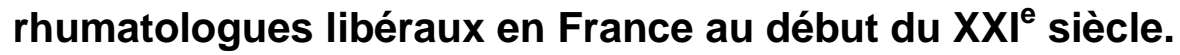

Comparaison aux recommandations EULAR 2006.

Julia Goossens ${ }^{1}$, Sylvie Lancrenon², Sabine Lanz ${ }^{3}$, Hang-Korng Ea ${ }^{1,4 ; 5}$, Charles Lambert $^{6}$, Pascal Guggenbuhl ${ }^{7}$, Alain Saraux ${ }^{8}$, Catherine Delva ${ }^{2}$, Pierre Chiarelli $^{9}$, Frédéric Lioté ${ }^{1,4,5}$

1. Service de rhumatologie, hôpital Lariboisière, AP-HP, Centre Viggo Petersen, 75010 Paris, France ;

2. Sylia-Stat, 92340 Bourg La Reine, France ;

3. Laboratoires Mayoly-Spindler, 78401 Chatou, France ;

4. Univ. Paris Diderot, Sorbonne Paris Cité, 75205 Paris, France ;

5. Inserm, UMR 1132, centre Viggo Petersen, 75010 Paris, France ;

6. Laboratoires Ipsen Pharma, Boulogne-Billancourt, 92100, France ;

7. CHU Rennes, service de rhumatologie, 35203 Rennes, France ;

8. INSERM UMR 991, 35000 Rennes, France ;

9. Université Rennes 1, Faculté de médecine, 35043 Rennes, France ; 10. Université de Bretagne occidentale; $\mathrm{CHU}$ de la Cavale Blanche, service de Rhumatologie, 29000 Brest, France ;

11. Vivactis Études Cliniques, 92400 Courbevoie, France.

Correspondance : Frédéric Lioté

Service de Rhumatologie, Centre Viggo Petersen, hôpital Lariboisière (AP-HP), 2 , rue Ambroise Paré, F-75010 Paris, France

Tel : +33149956291

Fax : +33149958631

Mail : frederic.liote@aphp.fr 


\section{Résumé}

Introduction. En 2006, des recommandations de prise en charge de la goutte ont été élaborées par l'EUropean League Against Rheumatism (EULAR). Le but de ce travail était de les comparer à la prise en charge par des médecins libéraux.

Méthode. Dans cette étude prospective multicentrique nationale en France, des omnipraticiens et des rhumatologues libéraux ont été tirés au sort en 2009. Chacun a inclus deux patients goutteux consécutifs qui ont été évalués à deux reprises : lors de la consultation initiale puis entre les 3 et 6 mois suivants ; les informations sur les modalités de prise en charge EULAR 2006 ont été recueillies de façon standardisée. Résultats. Sur un total de 1003 patients, 771 patients ont été évalués aux 2 temps. L'allopurinol était prescrit chez $75,1 \%$ des patients. Lors de l'instauration de l'allopurinol $(n=44)$, seuls $43,2 \%$ d'entre eux ont reçu une dose initiale de $100 \mathrm{mg} / \mathrm{j}$. La prévention des accès par la colchicine a concerné $74,3 \%$ des patients. Sur 522 patients pour lesquels l'allopurinol est en cours à la consultation initiale, seuls $34,5 \%$ avaient une uricémie $\leq 360 \mu \mathrm{mol} / \mathrm{l}$ (dose moyenne : $173 \mathrm{mg} / \mathrm{j})$. Une alimentation trop riche a été repérée chez $31,5 \%$ des patients en surpoids ou obèses par les généralistes, et chez 19,7\% par les rhumatologues, suivie de conseils nutritionnels chez $45,8 \%$. La correction d'une consommation excessive d'alcool n'a été recommandée que dans $10 \%$ des cas. Les diurétiques n'ont été interrompus que chez 7 patients sur 175 possibles.

Conclusion. Des écarts aux recommandations EULAR 2006 ont été identifiés, posant la question de leur simplification et de leur implémentation dans la formation médicale initiale et continue. 
Mots-clés : goutte ; prise en charge ; colchicine ; traitement hypouricémiant ; allopurinol ; AINS ; diurétiques ; comorbidités ; insuffisance rénale ; inertie clinique. 


\section{Introduction}

La goutte est le rhumatisme inflammatoire le plus fréquent chez l'homme. Sa prévalence augmente avec l'âge et varie de 0,1\% à $10 \%$ dans le monde. En France métropolitaine, sa prévalence vient d'être estimée à 0,9 \% chez l'adulte [1].

Elle résulte d'une hyperuricémie chronique responsable de dépôts de microcristaux d'urate de sodium dans et autour des articulations et des os. L'accès aigu goutteux se manifeste par une ou plusieurs arthrites stéréotypées [2]. Les arthropathies chroniques peuvent donner lieu à des destructions ostéo-articulaires et une moindre qualité de vie. Elle est associée à l'obésité et à l'hyperinsulinisme, au diabète, aux maladies cardiovasculaires et à l'insuffisance rénale chronique (IRC). Or la sévérité de cette maladie est très souvent méconnue des patients mais aussi des soignants.

En 2006, des recommandations ont été élaborées par l'EUropean League Against $\boldsymbol{R}$ heumatism (EULAR) sur la prise en charge diagnostique et thérapeutique de la goutte[3,4] [Annexe A, Tableau S1; voir le matériel complémentaire accompagnant la version en ligne de cet article] : leur révision est attendue[5]. Depuis, d'autres pays ont émis des recommandations, comme l'American College of Rheumatology (ACR) en 2012 [6-9]. Leur mise en place n'a jamais fait l'objet de procédures d'implémentation spécifique.

En France, après la publication des recommandations EULAR 2006, leur dissémination n'a fait l'objet que d'articles de synthèse dans des revues de formation médicale continue $(F M C)$ en médecine générale $(M G)$ et en rhumatologie $(R H)$. Quatre pays européens ont ainsi évalué les écarts entre ces recommandations et leurs propres pratiques[10-12]. En France, l'étude GOSPEL 2 a permis d'évaluer le traitement de l'accès aigu en pratique libérale et de le comparer aux recommandations EULAR [13]. 
Le but de ce travail «GOSPEL 3 » a été de comparer la prise en charge pharmacologique, à l'exception de la colchicine[13], et non pharmacologique des patients goutteux en $M G$ et $\mathrm{RH}$ libérales, par rapport aux recommandations EULAR $2006[3,4]$.

\section{Méthodes}

\subsection{Description de l'étude}

La méthodologie précise de l'ensemble de GOSPEL est publiée [14]. Pour ce travail prospectif multicentrique national, des $M G$ et des $\mathrm{RH}$ ont été sélectionnés par tirage au sort. Chaque médecin devait inclure, après information et accord, deux patients goutteux consécutifs (Figure 1). Chaque patient a été évalué à deux reprises : lors de la consultation initiale, le jour de l'inclusion, et lors d'une seconde consultation ou d'un entretien téléphonique dans les trois à six mois suivants. Les caractéristiques de la population ont déjà été publiées [13] [Annexe A, Tableau S2] ;

1.2Données recueillies par le médecin

Les données suivantes ont été recueillies sur un cahier d'observation individuel (CRF) à la visite initiale :

- Recherche de facteurs de risques modifiables

- Mesures non pharmacologiques prises par le médecin

- Thérapeutiques : les traitements antérieurs et actuels de la goutte ont été colligés. Les questions sur les traitements hypouricémiants (THU) n'ont concerné que l'allopurinol, le probénécide et la benzbromarone, seules médications disponibles en France lors de l'étude (Octobre 2009).

- Concernant l'existence d'un THU, une goutte sévère a été définie selon la recommandation EULAR $n^{\circ} 7$ : nombre d'accès goutteux $\geq 3$, présence de tophus, arthropathie clinique et/ou radiographique chez les patients ayant une goutte chronique. 


\subsection{Données recueillies par le patient}

En fin de visite initiale, deux questionnaires d'auto-évaluation ont été remis à chaque patient, l'un à renvoyer dans les 15 jours suivant, l'autre trois à six mois plus tard.

Le premier auto-questionnaire comportait une partie sur l'activité physique, les habitudes de consommation (apports liquidiens, boissons alcoolisées et sucrées, alimentation), le tabagisme, des mesures pharmacologiques et non pharmacologiques. Le second auto-questionnaire cherchait à préciser les modifications du style de vie conseillées par le $\mathrm{MG} / \mathrm{RH}$ et effectuées par le patient pendant les 3-6 mois de suivi.

\subsection{Prescriptions en fin de consultation}

Il était demandé au praticien, à la fin de chaque consultation, si la prise en charge avait été modifiée, et en particulier si des médications avaient été arrêtées ou introduites (questions ouvertes). Les autres mesures non pharmacologiques ou sur le style de vie faisaient l'objet de questions ouvertes.

\section{5 Évaluation finale par le médecin}

Au total, 771 patients ont été évalués 3 à 6 mois après l'inclusion. Les patients ont été revus $(n=709)$ ou contactés par téléphone $(n=62)$ par leur médecin. Les informations suivantes ont été recueillies : nouveaux accès, consultations ayant pour motif la goutte, examens complémentaires réalisés, suivi du THU : maintien sans interruption (posologie, ajustement, observance, traitement préventif des crises) ou interruption (date, motif, caractère temporaire ou définitif), nouvelles prescriptions. 
Toutes les données statistiques ont été entrées sous SAS version 8.2 II s'agissait d'une simple analyse descriptive : variables qualitatives décrites en termes d'effectifs et pourcentages des différentes modalités de réponses et variables quantitatives décrites en termes d'effectifs, moyennes, écarts-types, médianes, valeurs extrêmes.

1.7. Sources de financement: soutien institutionnel des laboratoires MayolySpindler et Ipsen Pharma. Les représentants pharmaciens et médecins ont participé à la réflexion sur la méthodologie, l'analyse des résultats, la relecture critique de l'article. La soumission de ce manuscrit n'a pas été liée à un accord préalable des laboratoires.

\section{Résultats}

Les différents résultats sont à rapprocher des recommandations EULAR 2006 [Annexe A, Table S1]

2.1. Repérage des facteurs de risque modifiables par le médecin

\subsubsection{Alimentation trop riche}

Une alimentation «trop riche », non quantifiée, a été reconnue chez 29,3\% des patients suivis par les MG et chez $18,2 \%$ des patients suivis par les $\mathrm{RH}$, soit un total de $27,2 \%$ des patients. Une alimentation trop riche a été repérée chez $29,3 \%$ des patients suivis par les MG, tous IMC confondus, et chez seulement $31,5 \%$ des patients en surpoids ou obèses (IMC $>25 \mathrm{~kg} / \mathrm{m} 2$ ). Parmi les patients suivis en $\mathrm{RH}$, $18,2 \%$ des patients tous IMC confondus avaient une alimentation trop riche ; c'était le cas chez $19,7 \%$ des patients en surpoids ou obèses (IMC $\geq 25 \mathrm{~kg} / \mathrm{m} 2$ ).

\subsubsection{Consommation excessive d'alcool}


Une consommation excessive d'alcool a été repérée chez $15,1 \%$ des patients au total $(14,4 \%$ des patients en MG, $17,6 \%$ des patients en $\mathrm{RH})$. Chez les patients suivis en MG, lorsque la consommation excessive d'alcool (> $30 \mathrm{~g} / \mathrm{j}$ chez l'homme, $>20 \mathrm{~g} / \mathrm{j}$ chez la femme) était identifiée, elle était en moyenne de $56 \mathrm{~g} / \mathrm{j}( \pm 54 \mathrm{~g} / \mathrm{j})$. Chez les patients suivis en $\mathrm{RH}$, la consommation excessive d'alcool était moindre, $41 \mathrm{~g} / \mathrm{j}( \pm 24 \mathrm{~g} / \mathrm{j})$.

L'enquête directe par auto-questionnaire montre que $75 \%$ des patients ayant une consommation excessive d'alcool n'ont pas été identifiés spontanément par les MG et pour $71,2 \%$ des patients par les $\mathrm{RH}$.

La consommation de bière était faible, $6,4 \mathrm{~g} / \mathrm{j}( \pm 12,2 \mathrm{~g} / \mathrm{j})$ chez les hommes et de $1,5 \mathrm{~g} / \mathrm{j}( \pm 5,1 \mathrm{~g} / \mathrm{j})$ chez les femmes. Pour le vin et le champagne, elle était de $21,7 \mathrm{~g} / \mathrm{j}$ $( \pm 22,5 \mathrm{~g} / \mathrm{j})$ pour les hommes et de $6,9 \mathrm{~g} / \mathrm{j}( \pm 11,2 \mathrm{~g} / \mathrm{j})$ pour les femmes.

\subsection{Mesures prises notées par le médecin}

Des recommandations de modifier l'alimentation ont été proposées en fin de consultation par 45,8\% des médecins ( $46 \% \mathrm{MG}$ et $44,8 \% \mathrm{RH}$ ). Ce ne fut le cas que chez $47,9 \%$ des patients en surpoids ou obèses suivis en MG, et chez $38,8 \%$ des patients ayant un poids normal. Les $\mathrm{RH}$ l'ont noté pour $47,6 \%$ de leurs patients en surpoids ou obèses, et chez 36,4 \% de leurs patients de poids normal.

Une diminution de la consommation d'alcool a été proposée pour $8,1 \%$ des patients (MG : 8,4\% des patients, RH : 6,8\%). En MG, elle a été proposée pour des patients ayant une consommation d'alcool moyenne de $44,2 \mathrm{~g} / \mathrm{j}$ ( $\pm 23,4 \mathrm{SD})$, et chez $12,9 \%$ des patients ayant réellement une consommation excessive. En $\mathrm{RH}$, elle a été proposée pour des patients ayant une consommation d'alcool moyenne de $30 \mathrm{~g} / \mathrm{j}( \pm 17$ 
SD), et chez $10,9 \%$ des patients ayant réellement une consommation d'alcool excessive.

\subsection{Recherche des comorbidités (recommandation EULAR $n^{\circ} 3$ )}

L'hyperlipidémie touchait $46,5 \%$ des patients. Elle était traitée, ou l'ordonnance renouvelée, ou mentionnée chez $87,3 \%$ des patients au total, $90 \%$ par les MG et $70,8 \%$ par les $\mathrm{RH}$.

Un total de $14,9 \%$ des patients avait un diabète sucré. Ils étaient traités par une médication dans $83,8 \%$ des cas au total, dans $85,3 \%$ des cas en MG et dans $73,7 \%$ des cas en $\mathrm{RH}$.

L'HTA concernait 54,2\% des patients. Parmi ces malades $98,3 \%$ étaient traités (98,9\% pour les $M G$ et $95,9 \%$ pour les $R H)$.

Au total, $28 \%$ des patients seulement n'avaient ni HTA, ni diabète ou hyperlipidémie associés. À l'inverse, 35,3\% avaient une seule comorbidité, 29,2\% avaient deux comorbidités, et $7,5 \%$ en avaient trois. Parmi les patients ayant au moins une comorbidité, 12,8\% des patients avaient au moins une comorbidité non traitée, $11 \%$ des patients suivis par les $M G$ et $21,3 \%$ des patients suivis par les $R H$.

\subsection{Traitements de l'accès aigu}

\subsubsection{Traitements habituels de l'accès aigu}

Les données GOSPEL 2 sur la colchicine sont publiées [12]. Les traitements habituels de l'accès aigu sont résumés dans la figure 2. II s'agissait de la colchicine seule, de la colchicine associée à un ralentisseur du transit $\left(\right.$ Colchimax $\left.^{\circledR}\right)$, des anti- 
inflammatoires non stéroïdiens (AINS), de la corticothérapie orale ou des infiltrations intra articulaires de corticoïdes.

\subsubsection{Traitement par colchicine}

La dose utilisée au premier jour de traitement était en moyenne de $3 \mathrm{mg}( \pm 0,5 \mathrm{SD})$ en MG, et de $2,7 \mathrm{mg}( \pm 0,7 \mathrm{SD})$ en $\mathrm{RH}$. Cette dose de $3 \mathrm{mg}$ était la dose la plus fréquemment prescrite, que ce soit par les $M G$ ou les $\mathrm{RH}$ ( $86,1 \%$ des cas au total). Le schéma posologique le plus fréquemment utilisé pour les 7 premiers jours par les MG et les RH était le schéma ancien : 3 mg-2 mg-2 mg-1 mg pour les jours J1-J2-J3J4 à J7. La durée moyenne du traitement a été de 16,5 jours pour les MG $( \pm 20,0 S D)$ et de 21,4 jours pour les $\mathrm{RH}( \pm 36,9 \mathrm{SD})$.

2.4.3 Adaptation du traitement de l'accès aigu en fonction de la clairance de la créatinine

Cet aspect dérivé des recommandations d'emploi des AINS est essentiel à prendre en compte du fait de la prévalence de l'insuffisance rénale chronique de niveau 3 et de sa sévérité chez les goutteux. Nous avons utilisé la formule de Cockcroft-Gault pour estimer le débit de filtration glomérulaire (DFGe).

Chez les MG, les AINS ont été prescrits à $26,4 \%$ des patients avec une fonction rénale normale (DFGe $\geq 80 \mathrm{ml} / \mathrm{min}$ ) et à $36 \%$ des patients ayant une insuffisance rénale (IRC) légère (DFGe 60-80 $\mathrm{ml} / \mathrm{min}$ ). Toutefois $16 \%$ des patients ayant une IRC modérée (IRC 3, DFGe 30-60 ml/min), et la moitié des patients atteints d'une IRC sévère (IRC 4, DFGe 15-30 ml/min) ont reçu une prescription d'AINS. 
Chez les $\mathrm{RH}$, des AINS ont été prescrits à la moitié de patients qui n'avaient pas d'insuffisance rénale, à $54,5 \%$ des patients qui avaient une réduction fonctionnelle légère, et à $26,7 \%$ des patients qui avaient une IRC 3 . Ils n'ont pas été prescrits au seul patient atteint d'IRC 4 ou terminale (clairance $<15 \mathrm{ml} / \mathrm{min}$ ).

\subsection{Traitement hypouricémiant au long cours}

Les patients avaient un THU par allopurinol en cours à l'inclusion dans $2 / 3$ des cas ( $67,5 \%$ des cas dont $67,3 \%$ en MG et $68,4 \%$ en $\mathrm{RH}$ ) (Tableau 1). Ce THU était présent dans les antécédents chez 16,1\% des patients (17,2\% MG et 11,6\% pour $\mathrm{RH})$. Il a été instauré à la fin de la première consultation pour $5,3 \%$ des patients (4,9\% MG et 7,4\% RH). L'allopurinol n'a jamais été prescrit chez $11 \%$ des patients (10,6\% MG, $12,6 \% R H)$.

Le probénécide était en cours chez $3,1 \%$ des patients ( $3 \% \mathrm{MG}$ et $3,9 \% \mathrm{RH}$ ). II a été nouvellement prescrit chez un seul patient $(\mathrm{RH})$.

\section{Au total, $\mathbf{7 5 , 1} \%$ des patients recevaient un THU (allopurinol ou probénécide)} ( $73,8 \%$ pour les MG, $80,3 \%$ pour les $\mathrm{RH})$.

L'existence d'un THU a été étudiée en fonction de la sévérité de la goutte (cf Méthodes). Une goutte sévère concernait $81,1 \%$ des patients, $80,9 \%$ en MG et $81,9 \%$ en $\mathrm{RH}$. Cependant seuls $77,9 \%$ des patients ayant une goutte sévère avaient ou ont eu une prescription de THU (76,2\% en MG, 84,6 \% en $\mathrm{RH})$.

\subsection{Dosage de l'uricémie}


Un dosage de l'uricémie datant de moins d'un an et son résultat disponible dans les dossiers ont été notés par les praticiens pour 78,5 \% des patients (77\% MG, 84,8 \% $\mathrm{RH})$.

Les patients traités par allopurinol avaient une uricémie moyenne de $403 \mu \mathrm{mol} / \mathrm{l}$ ( $\pm 97 S D)$ en $M G$, et $398 \mu \mathrm{mol} / /( \pm 95 S D)$ en $\mathrm{RH}$. Moins d'un tiers des patients avait une uricémie inférieure à la valeur cible : 28,0\% des patients suivis en MG, et 29,3\% des patients suivis en $\mathrm{RH}$.

L'allopurinol a été nouvellement prescrit chez 44 patients (en MG, patients avec uricémie moyenne de $481 \mu \mathrm{mol} / \mathrm{l}( \pm 85 \mathrm{SD})$ et en $\mathrm{RH}$, patients avec uricémie moyenne de $513 \mu \mathrm{mol} / /( \pm 39 S D))$.

\subsection{Adaptation de la posologie initiale d'allopurinol}

Chez 44 patients, l'allopurinol a été débuté en fin de consultation. La dose initiale prescrite était en moyenne de $171 \mathrm{mg}$ ( $\pm 69 \mathrm{SD}$ ) chez les MG et $177 \mathrm{mg}$ ( $\pm 83 \mathrm{SD}$ ) chez les $\mathrm{RH}$. Au total, $58 \%$ des patients suivis par les MG ont reçu une dose initiale supérieure à 100 mg, et 53,8 \% pour ceux suivis par les $\mathrm{RH}$. (Figure 3A)

L'adaptation de posologie en fonction du DFGe est décrite dans la figure 3B.

La variation de posologie d'allopurinol entre la première et la dernière visite, chez les patients ayant maintenu leur traitement, est décrite figure 4.

\subsection{Arrêt de l'allopurinol}

L'allopurinol a été arrêté chez $26,3 \%$ des patients traités pour des motifs variés, en particulier d'une décision du patient dans $72,4 \%$ des cas (76,2\% MG et $52,8 \% \mathrm{RH})$. 
Les autres raisons sont les suivantes: a) normalisation de l'uricémie dans $15,7 \%$ des cas $(18,2 \% \mathrm{MG}, 2,8 \% \mathrm{RH})$, b) Intolérance dans $11,1 \%$ des cas $(6,1 \% \mathrm{MG}$, $36,1 \% \mathrm{RH}$; aucun cas de toxidermie sévère type syndrome de Lyell ou de DRESS) «Manque d'efficacité » dans 2,3\% des cas (1,7\% MG, 5,6\% en RH).

\subsection{Traitement préventif à l'instauration de l'allopurinol}

Les patients étant (ou ayant déjà été) traités par THU ont reçu un traitement préventif de l'accès aigu à l'instauration de l'allopurinol dans $74,3 \%$ des cas $(72,7 \%$ en $M G$, $81,9 \%$ en $\mathrm{RH})$. II s'agissait de la colchicine dans $98,2 \%$ des cas $(98,8 \% \mathrm{MG}$, $95,9 \% R H)$.

Chez les 44 patients nouvellement traités la colchicine a été prescrite pour une durée moyenne de 7,7 semaines $( \pm 4,7 \mathrm{SD})$ en $M G$ et 11,2 semaines $( \pm 7,8 S D)$ en $R H$. Cette prescription associée de colchicine $(1 \mathrm{mg} / \mathrm{j})$ n'a concerné que $55 \%$ des cas $(37,9 \%$ en $\mathrm{MG}, 100 \%$ en $\mathrm{RH})$.

\subsection{Prise de diurétiques}

Un traitement par diurétique(s) figurait comme co-prescription chez $22,9 \%$ des patients : $23 \%$ des patients suivis en MG et $22,2 \%$ des patients suivis en $\mathrm{RH}$. II s'agissait d'un traitement anti-HTA dans $88,6 \%$ des cas, et comme traitement de l'insuffisance cardiaque dans $11,4 \%$ des cas. Ce traitement n'a été identifié par le médecin comme facteur de risque modifiable que dans $23 \%$ des cas par les MG et $22,5 \%$ des cas par les $\mathrm{RH}$. En l'absence d'insuffisance cardiaque, le diurétique n'a été arrêté que chez 7 de 175 patients (4,5\%) pour les MG. II n'y a pas eu de recommandations d'arrêt de diurétiques par les $\mathrm{RH}$. 


\section{Discussion}

L'étude GOSPEL est la première étude descriptive sur les pratiques de prise en charge de la goutte en France par les médecins généralistes et les rhumatologues libéraux. Elle permet ici de décrire les pratiques de prise en charge thérapeutique entre les médecins confrontés à la goutte en France en 2009, et de les comparer avec les recommandations de référence. Des décalages dans la pratique par rapport à celles-ci ont été identifiés, en particulier :

- moins d'un patient sur 2 nouvellement traité a reçu une dose initiale d'allopurinol de $100 \mathrm{mg} / \mathrm{j}$

- un faible taux de patients goutteux avait à 6 mois une uricémie à la cible $<360$ $\mu \mathrm{mol} / \mathrm{I}(29,3 \%$ des patients suivis en $\mathrm{RH}$, et $28 \%$ pour ceux suivis en $M G)$, avec une inertie à augmenter la posologie du THU puisque la posologie maximale moyenne était de $173 \mathrm{mg} / \mathrm{j}$.

- À l'inverse, on a pu relever une assez large prescription de THU $(75,1 \%$ des patients ; $73,8 \%$ en $M G, 80,3 \%$ en $\mathrm{RH}$ ) et la prévention des accès par la colchicine chez la quasi-totalité des patients.

Une des premières recommandations EULAR atteste de l'importance de la prise en charge des facteurs de risque modifiables [4]. Ces facteurs de risque ne sont pas toujours connus ou repérés par les soignants, et lorsqu'ils le sont, ils ne sont pas toujours pris en charge. Une alimentation trop riche, terme sans doute imprécis, n'a 
été repérée que chez $31,5 \%$ des patients en surpoids ou obèses par les MG, et chez $19,7 \%$ chez les RH. Elle n'a été prise en charge que chez $45,8 \%$ des patients. On sait pourtant que la réduction de poids s'accompagne d'une réduction linéaire de l'uricémie[15]. À ce titre il est utile de rechercher systématiquement le surpoids en calculant I'IMC pour débuter ces conseils nutritionnels utiles également dans le diabète, l'HTA et la dyslipidémie [16].

En 2006, certains facteurs nutritionnels n'avaient pas encore été identifiés, comme le fructose des sodas sucrés[17,18]. Ils figurent désormais dans les recommandations ACR 2012 [6,7] et dans les recommandations EULAR actualisées [5]. II existe une interaction entre IMC et consommation de sodas sucrés favorisant le défaut d'excrétion urinaire d'acide urique[19].

L'excès d'alcool est un élément important et simple à quantifier. Dans ce travail, une consommation excessive d'alcool était présente chez $54 \%$ des patients, mais sa correction a rarement été recommandée (10\% des cas environ). Cette démarche quantitative est pertinente car il s'agit d'apports caloriques inutiles (90 à 150 calories par unité de boissons, soit $10 \mathrm{~g}$ d'alcool[20].Une démarche qualitative doit aussi être mise en œuvre à la suite de travaux épidémiologiques associant la consommation de bière à l'hyperuricémie et à la goutte[21]. Les recommandations ACR ne vont que dans le sens d'une réduction de la consommation de bière $[7,8]$ alors que pour les Européens, cette consommation doit être interrompue, y compris pour les bières sans alcool[6]. Les alcools durs doivent aussi être interrompus non tant pour les apports caloriques que pour d'autres mécanismes favorisant l'hyperuricémie[21].

La recherche de comorbidités est un élément clé dans la prise en charge des patients goutteux, en particulier en MG. Seuls $28 \%$ d'entre eux n'avaient aucune 
comorbidité. Si les dyslipidémies et le diabète de type II sont en général bien pris en charge (traités respectivement dans $87,3 \%$ et $83,8 \%$ des cas), I'HTA n'est apparemment traitée que dans la moitié des cas ; il peut s'agir aussi de patients n'ayant pas encore d'HTA permanente ou seulement traités par des mesures diététiques. II apparaît que la goutte est associée à un sur risque de mortalité cardio vasculaire, d'infarctus du myocarde et d'AVC[22], d'où un raisonnement à double sens : rechercher les comorbidités chez un patient goutteux.

En cas de crise, la colchicine était plus fréquemment utilisée que les AINS [23]. Les doses prescrites étaient souvent supérieures aux recommandations[13], exposant aux risques d'effets indésirables. À la suite de l'essai pivot nord-américain en 2010 [24], la dose moyenne de colchicine recommandée aux USA le premier jour est désormais plus faible (1,8 $\mathrm{mg}$ à $\mathrm{J} 1)$, ce qui permet une efficacité identique aux doses élevées utilisées aux USA (4,8 mg J1) ou même en France (3 mg J1 au maximum en 2016) avec une meilleure observance et moins d'effets indésirables [4].

Quel que soit le traitement de l'accès envisagé, colchicine ou AINS, il est nécessaire de disposer au moins une fois par an d'une estimation de la fonction rénale par une formule adaptée au sexe, à l'âge (CKD EPI, MDRD) et éventuellement à l'ethnie (MDRD). Dans la vraie vie cette mesure manque régulièrement. Cette information n'a pas été recueillie dans notre travail.

Le principal objectif du traitement est la prescription d'un THU. Dans cette cohorte GOSPEL plus de $75 \%$ des patients avaient une prescription de THU, fréquence supérieure aux autres séries [9-12]. Les posologies des THU étaient souvent inadaptées à l'uricémie-cible. Ils ont été souvent prescrits à doses élevées ou maximales d'emblée, sans augmentation progressive de la posologie. Dans cette 
cohorte, seules $43 \%$ des prescriptions initiales d'allopurinol étaient de $100 \mathrm{mg} / \mathrm{j}$, témoignant d'un mésusage dans les autres cas.

L'absence d'adaptation à la fonction rénale du patient peut être à l'origine de problèmes de tolérance (toxidermie à l'allopurinol[25]). À l'inverse, dans cette cohorte, en l'absence d'ajustement d'augmentation de posologie, l'uricémie cible n'a été atteinte que chez $28 \%$ des patients ( $28 \%$ MG, 29,3\% RH). Ainsi la dose moyenne d'allopurinol était de $173 \mathrm{mg} / \mathrm{j}$, largement insuffisante pour abaisser l'uricémie.

Ces posologies moyennes modestes et insuffisamment efficaces traduisent une véritable inertie clinique [26] ou une crainte infondée d'un risque iatrogène[27]. Par ailleurs, lorsque le traitement de fond est arrêté, il l'est le plus souvent par le patient lui-même, pour une « mauvaise bonne raison », la normalisation de l'uricémie.

Depuis les recommandations EULAR 2006, de nouvelles thérapeutiques ont vu le jour, comme la pégloticase, indisponible en Europe, et le febuxostat[28], second THU inhibant la xanthine oxydase, commercialisé en France depuis Mars 2010. Son apport à la prise en charge de la goutte a été évalué en France en 2012 dans l'étude CACTUS[29]. Son principal intérêt est son efficacité qui se maintient en cas d'IRC 3 où l'augmentation des posologies d'allopurinol n'est pas recommandée en France[30]. La prise en charge des patients évolue et de nouvelles recommandations nord-Américaines[7,8] et Européennes[5] ont donc été adaptées à ces nouveaux traitements.

Les limites de ce travail sont liées à un effectif de 740 CRFs exploitables sur l'effectif initial de 1003 patients, ce qui représente $77 \%$ du total, essentiellement du fait de 
l'absence de réponse des patients aux deux questionnaires ou de l'absence de suivi dans le délai des 6 mois. Les résultats descriptifs peuvent être décevants mais aucune analyse statistique n'avait été prévue a priori per protocole. Certaines données dépendaient seulement du patient mais le taux de réponse et de retour a été très satisfaisant. La qualité du travail tient au caractère prospectif des données collectées et à leur recueil durant et en fin de consultation, ce qui constitue une des rares études internationales.

La goutte est une maladie pour laquelle des traitements efficaces sont disponibles. Toutefois, plus que le médicament lui-même, c'est surtout la manière de prendre en charge le patient par son information, certains diront l'éducation, la bonne adaptation des doses tant que l'uricémie n'est pas abaissée en dessous de valeurs cibles prédéfinies (« treat-to-target »), leur suivi régulier qui vont compter. Cela peut se faire au besoin par une infirmière clinicienne comme dans certains pays européens [31]. Dans le travail préliminaire de l'équipe de Doherty, $94 \%$ des patients étaient à l'uricémie-cible de $60 \mathrm{mg} / \mathrm{l}$, et $84 \%$ en dessous de $50 \mathrm{mg} / \mathrm{l}$ pour une dose médiane de $400 \mathrm{mg}$ d'allopurinol. Cette étude pilote est actuellement suivie d'une étude randomisée contrôlée en MG. La fréquence des crises de goutte, souvent seule ressentie du patient (et du médecin), était également à la baisse à la fin de l'année de suivi [29].

Ces problèmes de prise en charge ne sont pas une exception française, comme peuvent le montrer des études similaires notamment en Angleterre[12] ou aux EtatsUnis[32]. Les groupes d'éducation thérapeutique (ETP) sont une solution a priori efficace comme dans le diabète sucré[33], pour améliorer les connaissances des patients sur leur maladie et sur les thérapeutiques et l'adhésion aux traitements. 
Deux actions d'ETP goutte ont été mises en place en France[34] mais à ce jour aucun résultat en termes d'observance et de réponse clinico-biologique n'a été publié.

Le corollaire immédiat n'est pas tant le développement ou la mise à jour de recommandations, qu'à leur implémentation dans le cursus des études médicales et de la formation des médecins qualifiés, FMC incluse. On peut aussi proposer la simplification de recommandations souvent complexes pour un praticien occupé [35]. Une stratégie que nous soutenons réside dans la rédaction d'indicateurs de qualité, moyen de résumer de façon pertinente et concise de bonnes pratiques cliniques. Cela pourrait aussi faire l'objet d'incitations administratives et/ou financières, comme cela a déjà été fait en France pour le diabète sucré, par exemple, avec la régularité des mesures trimestrielles de l'HbA1c. 
Remerciements : Nous remercions le Dr Marie-Christine Andro-Delestrain (LGV, Mayolly-Spindler) et le $\operatorname{Pr}$ Jean-Pierre Aubert, Département de médecine générale, Université Paris Diderot, pour leur soutien et leur contribution au projet, et Mr Samy Sahbane (Vivactis Études Cliniques, Courbevoie) pour son travail de préparation, de mise en place et de contrôle qualité de l'étude. Les remerciements vont aussi aux médecins généralistes et rhumatologues qui ont participé au recueil des données et à leurs malades volontaires.

Déclaration de conflits d'intérêt : F. Lioté : honoraires de conseils ou d'expertises, participation à des sessions de FMC en rapport avec le thème : Astra-Zeneca, Ipsen, Menarini France et Global, Mayoly-Spindler, Novartis France, Novartis Global ; dons à visée d'enseignement pour l'organisation de Workshops sur les microcristaux et les maladies humaines : Ardea, Astra-Zeneca, Ipsen Pharma, Mayoly-Spindler, Ménarini France, Menarini international, Novartis France, Novartis Global, SOBI BioVitrum (www.european-crystal-network.com) ;

S. Lanz, employée des laboratoires Mayoly-Spindler ;

P. Guggenbuhl : intervention ponctuelle pour les laboratoires Ipsen et Menarini ;

C. Lambert : employé du laboratoire Ipsen Pharma ;

P. Chiarelli, S. Lancrenon, C. Delva : financement Mayoly-Spindler ;

A. Saraux, J.-P. Aubert, H.-K Ea et J. Goossens : aucun conflit d'intérêt. 


\section{Références}

[1] Kuo C-F, Grainge MJ, Zhang W, Doherty M. Global epidemiology of gout: prevalence, incidence and risk factors. Nat Rev Rheumatol 201;11:649-62.

[2] Burbage G. Gout: clinical presentation and management. Nurs Stand 2014;29:50-6.

[3] Zhang W, Doherty M, Pascual E, Bardin T, Barskova V, Conaghan P, et al. EULAR evidence based recommendations for gout. Part I: Diagnosis. Report of a task force of the Standing Committee for International Clinical Studies Including Therapeutics (ESCISIT). Ann Rheum Dis 2006;65:1301-11.

[4] Zhang W, Doherty M, Bardin T, Pascual E, Barskova V, Conaghan P, et al. EULAR evidence based recommendations for gout. Part II: Management. Report of a task force of the EULAR Standing Committee for International Clinical Studies Including Therapeutics (ESCISIT). Ann Rheum Dis 2006;65:1312-24.

[5] Richette $P$, Doherty $M$, Pascual $E$, et al. Updated EULAR evidence-based recommendations for the management of gout. Ann Rheum Dis 2014; 73:783

[6] Cottrell E, Crabtree V, Edwards JJ, Roddy E. Improvement in the management of gout is vital and overdue: an audit from a UK primary care medical practice. BMC Fam Pract 2013;14:170.

[7] Khanna D, Fitzgerald JD, Khanna PP, Bae S, Singh MK, Neogi T, et al. 2012 American College of Rheumatology guidelines for management of gout. Part 1: systematic nonpharmacologic and pharmacologic therapeutic approaches to hyperuricemia. Arthritis Care Res 2012;64:1431-46.

[8] Khanna D, Khanna PP, Fitzgerald JD, Singh MK, Bae S, Neogi T, et al. 2012 American College of Rheumatology guidelines for management of gout. Part 2: therapy and antiinflammatory prophylaxis of acute gouty arthritis. Arthritis Care Res 2012;64:1447-61.

[9] Manara M, Bortoluzzi A, Favero M, Prevete I, Scirè CA, Bianchi G, et al. Italian Society of Rheumatology recommendations for the management of gout. Reumatismo 2013;65:421.

[10] Owens D, Whelan B, McCarthy G. A survey of the management of gout in primary care. Ir Med J 2008;101:147-9.

[11] Pérez Ruiz F. [Treatment of Gout: Reflections From the GEMA Study]. Reumatol Clin 2008;4 Suppl 2:41-4.

[12] Roddy E, Zhang W, Doherty M. Concordance of the management of chronic gout in a UK primary-care population with the EULAR gout recommendations. Ann Rheum Dis 2007;66:1311-5.

[13] Pascart T, Lancrenon S, Lanz S, et al. GOSPEL 2: Colchicine for the treatment of gout flares. A GOSPEL Survey subgroup analysis. Doses used in common practices regardless of renal impairment and age. Joint Bone Spine 2016;83:687-93.

[14] Lioté F, Lancrenon S, Lanz S, Guggenbuhl P, Lambert C, Saraux A, et al. GOSPEL: prospective survey of gout in France. Part I: design and patient characteristics $(n=1003)$. Jt Bone Spine Rev Rhum 2012;79:464-70.

[15] Dalbeth N, Chen P, White M, Gamble GD, Barratt-Boyes C, Gow PJ, et al. Impact of bariatric surgery on serum urate targets in people with morbid obesity and diabetes: a prospective longitudinal study. Ann Rheum Dis 2014;73:797-802.

[16] Lam DW, LeRoith D. Metabolic Syndrome. In: De Groot L, Beck-Peccoz P, Chrousos G, Dungan K, Grossman A, Hershman JM, et al., editors. Endotext, South Dartmouth (MA): MDText.com, Inc.; 2000.

[17] Choi HK, Curhan G. Soft drinks, fructose consumption, and the risk of gout in men: 
prospective cohort study. BMJ 2008;336:309-12.

[18] Choi JW, Ford ES, Gao X, Choi HK. Sugar-sweetened soft drinks, diet soft drinks, and serum uric acid level: the Third National Health and Nutrition Examination Survey. Arthritis Rheum 2008;59:109-16.

[19] Dalbeth N, Phipps-Green A, House ME, Gamble GD, Horne A, Stamp LK, et al. Body mass index modulates the relationship of sugar-sweetened beverage intake with serum urate concentrations and gout. Arthritis Res Ther 2015;17:263.

[20] Food Surveys : What's In The Foods You Eat <em>Search Tool</em>, 2011-2012 n.d. http://www.ars.usda.gov/Services/docs.htm?docid=17032 (accessed November 1, 2015).

[21] Roddy E, Choi H. Epidemiology of Gout. Rheum Dis Clin North Am 2014;40:155-75.

[22] Borghi C, Rosei EA, Bardin T, Dawson J, Dominiczak A, Kielstein JT, et al. Serum uric acid and the risk of cardiovascular and renal disease. J Hypertens 2015;33:1729-41; discussion 1741.

[23] Lioté F, Bardin T. Traitement de la goutte. Rev Rhum 2007;74:160-7.

[24] Khanna PP, Gladue HS, Singh MK, et al. Treatment of acute gout: a systematic review. Semin Arthritis Rheum 2014;44:31-8.

[25] Kahn M-F, Bardin T, Dieudé $P$, et al. L'actualité rhumatologique 2013. Issy les Moulineaux : Elsevier Masson 2013.

[26] Lioté F, Choi H. Managing gout needs more than drugs: "Il faut le savoir-faire, I"Art et la manière'. Ann Rheum Dis 2013;72:791-3.

[27] Dalbeth N, Stamp LK. Gout: Why compare the effectiveness of suboptimal gout management? Nat Rev Rheumatol 2015;11:506-7.

[28] Richette P, Ottaviani S, Bardin T. [New therapeutic options for gout]. Presse Médicale Paris Fr 1983 2011;40:844-9.

[29] Richette P, Flipo RN, Patrikos DK. Characteristics and management of gout patients in Europe: data from a large cohort of patients. Eur Rev Med Pharmacol Sci 2015;19:630-9.

[30] Richette P, Frazier A, Bardin T. Pharmacokinetics considerations for gout treatments. Expert Opin Drug Metab Toxicol 2014;10:949-57.

[31] Rees F, Jenkins W, Doherty M. Patients with gout adhere to curative treatment if informed appropriately: proof-of-concept observational study. Ann Rheum Dis 2013;72:82630.

[32] Oderda GM, Shiozawa A, Walsh M, Hess K, Brixner DI, Feehan M, et al. Physician adherence to ACR gout treatment guidelines: perception versus practice. Postgrad Med 2014;126:257-67. .

[33] Renda S, Baernholdt M, Becker K. Evaluation of a Worksite Diabetes Education Program at a Large Urban Medical Center. Workplace Health Saf 2016;64:17-23.

[34] A. Frazier-Mironer, O. Peyr, HK. Ea, F. Lioté, Richette P, T. Bardin. Efficacité de l'éducation thérapeutique (ETP) dans la goutte : résultats sur 80 patients. Revue du Rhumatisme 2016; 83(suppl.1):A13

[35] Bardin T, Richette P. New ACR guidelines for gout management hold some surprises. Nat Rev Rheumatol 2013;9:9-11. 


\section{Légendes des figures}

Figure 1 : Schéma d'analyse ("flow chart») de la cohorte GOSPEL - analyse GOSPEL 3 disposant des deux consultations, à l'inclusion et au suivi entre 3 et 6 mois $(n=771)$.

Figure 2. Description des modalités de traitement d'un accès aigu - analyse basée sur la population GOSPEL $1(\mathrm{n}=978)$.

Figure 3. Nouvelles prescriptions d'allopurinol ( $n=44$ patients) - comparaison à la recommandation EULAR $n^{\circ} 9$. A. Posologies initiale et finale d'allopurinol ; B. Adaptation de dose à la fonction rénale estimée selon la formule de Cockroft et Gault. MG : médecins généralistes; RH : rhumatologues libéraux.

Figure 4. Variations de la posologie journalière d'allopurinol entre la première et la seconde consultation ( $n=590$ malades encore sous traitement). Exprimée en $\mathrm{mg} / \mathrm{j} . \mathrm{d}$ 
Tableau 1 : Patients traités par allopurinol dits « à la cible » $(\leq 360 \mu \mathrm{mol} / \mathrm{l})$ lors du dernier dosage annuel d'uricémie (\%)

\begin{tabular}{|l|c|c|}
\hline & $\begin{array}{c}\text { Patients suivis par les MG } \\
(\mathrm{n}=416)\end{array}$ & $\begin{array}{c}\text { Patients suivis par les RH } \\
(\mathrm{n}=106)\end{array}$ \\
\hline Patients à la cible & $32,7 \%(136)$ & $\mathbf{4 1 , 5 \% ( 4 4 )}$ \\
\hline Patients au-dessus de la cible & $\mathbf{6 7 , 3 \% ( 2 8 0 )}$ & $\mathbf{5 8 \% ( 6 2 )}$ \\
\hline Uricémie moyenne $(\mu \mathrm{mol} / \mathrm{l})$ & 403 & 398 \\
\hline
\end{tabular}


Tableau 1 : Patients traités par allopurinol dits « à la cible » $(\leq 360 \mu \mathrm{mol} / \mathrm{l})$ lors du dernier dosage annuel d'uricémie (\%)

\begin{tabular}{|l|c|c|}
\hline & $\begin{array}{c}\text { Patients suivis par les MG } \\
(\mathrm{n}=416)\end{array}$ & $\begin{array}{c}\text { Patients suivis par les RH } \\
(\mathrm{n}=106)\end{array}$ \\
\hline Patients à la cible & $32,7 \%(136)$ & $41,5 \%(44)$ \\
\hline Patients au-dessus de la cible & $\mathbf{6 7 , 3 \% ( 2 8 0 )}$ & $\mathbf{5 8 , 5 \% ( 6 2 )}$ \\
\hline Uricémie moyenne $(\mu \mathrm{mol} / \mathrm{l})$ & 403 & 398 \\
\hline
\end{tabular}




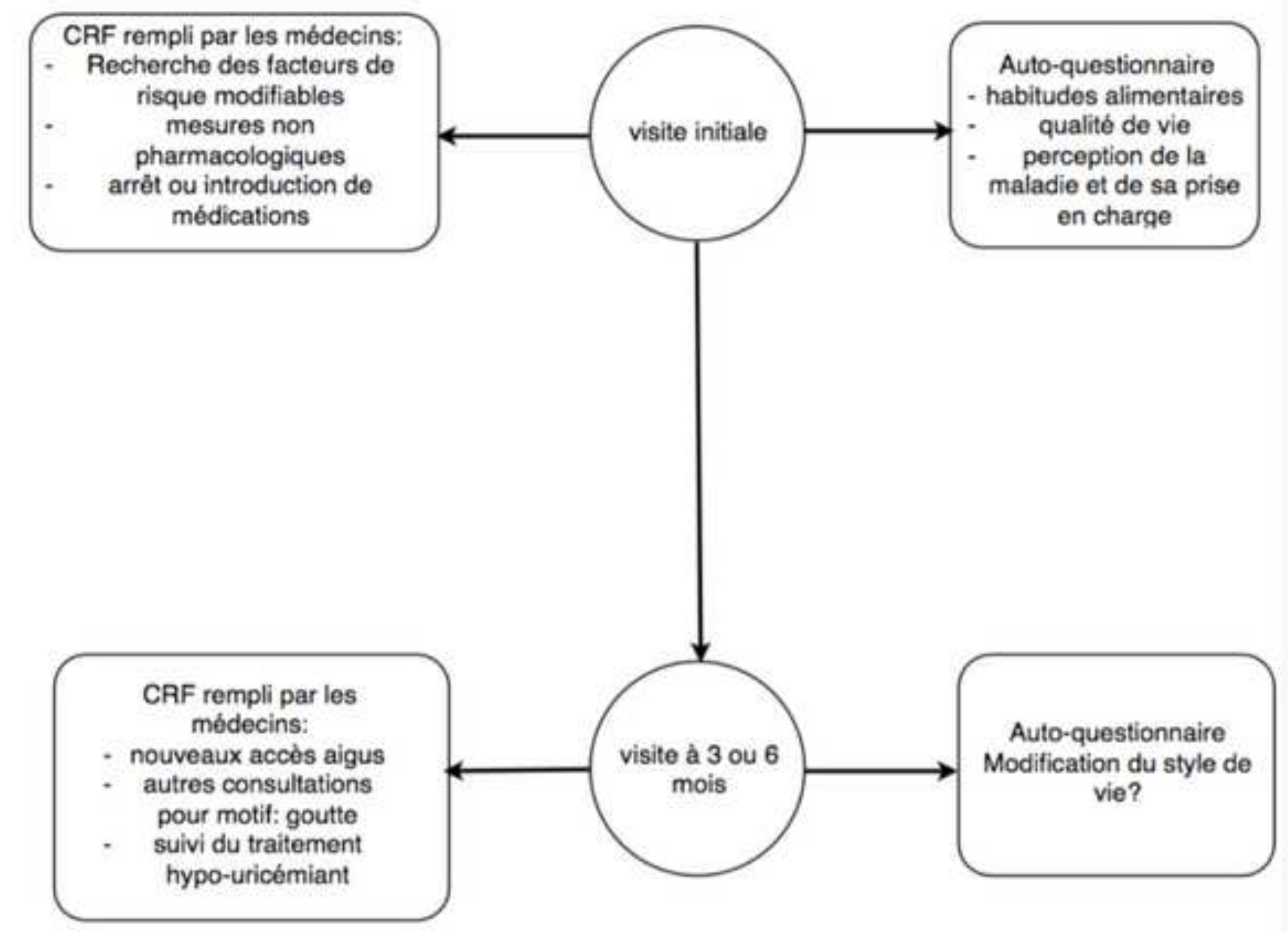

\section{CRF : cahier d'observation individuel}




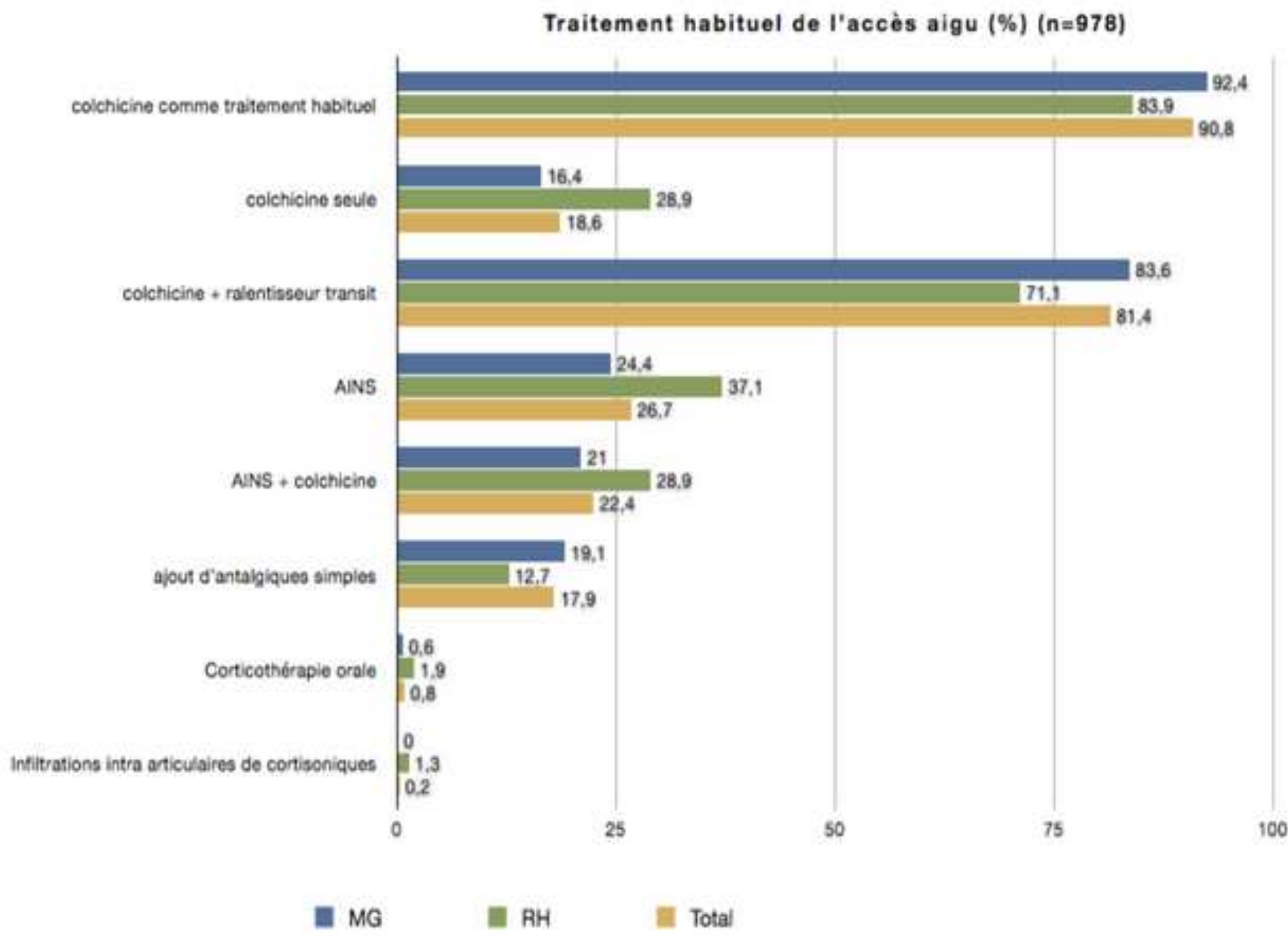
a MG
1. $\mathrm{RH}$
Total 
Dose initiale prescrite d'allopurinol pour les patients nouvellement traités $(n=44)$ $100 \%$

$75 \%$

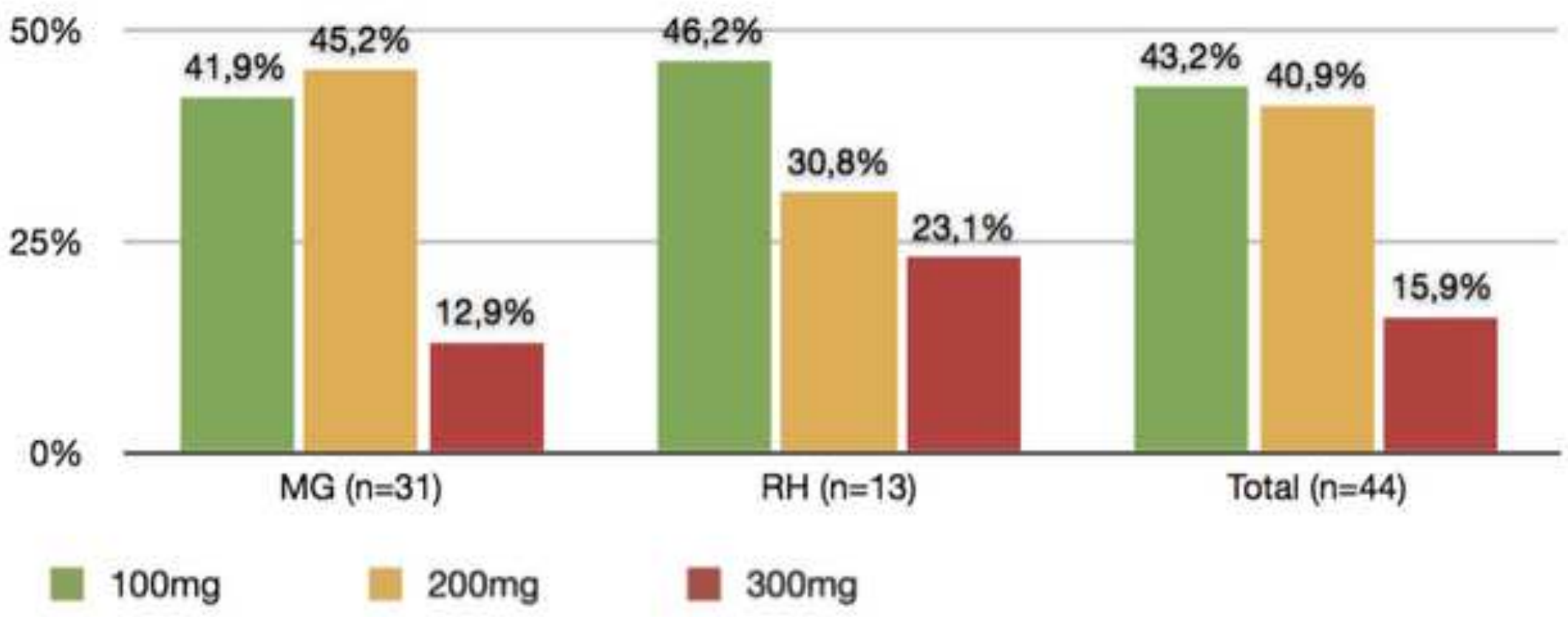




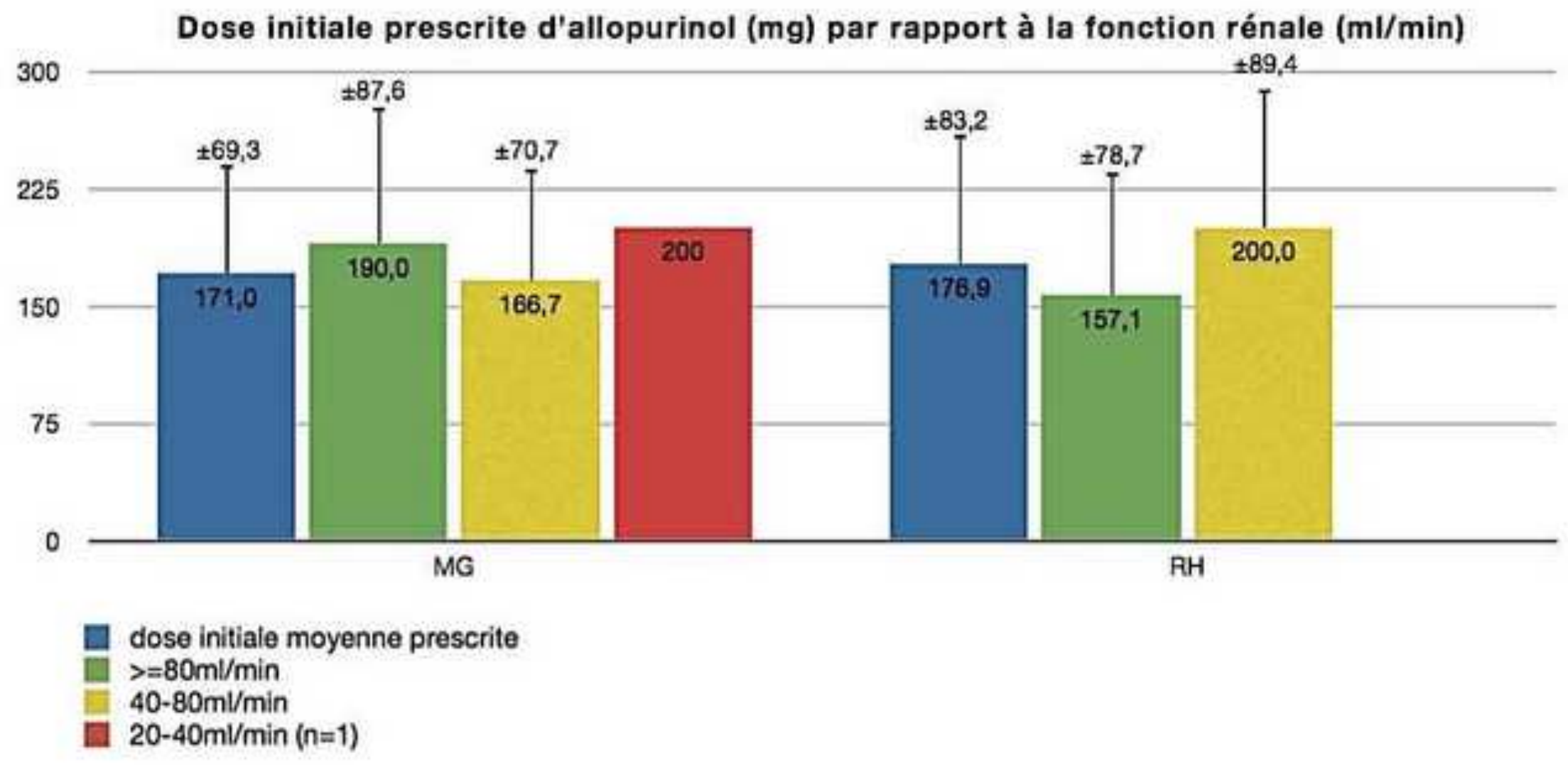


Variation de la posologie d'allopurinol $(\mathrm{mg})$ entre la première et la dernière visite chez les patients ayant maintenu leur traitement ( $\mathrm{n}=590$ )

500

375

250

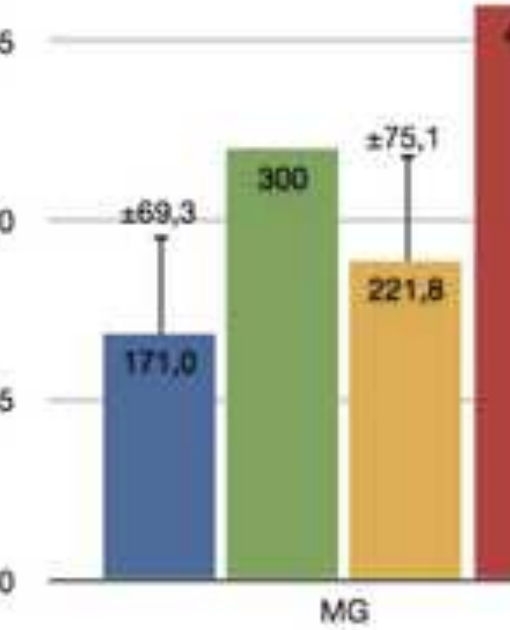

0

\section{s00}
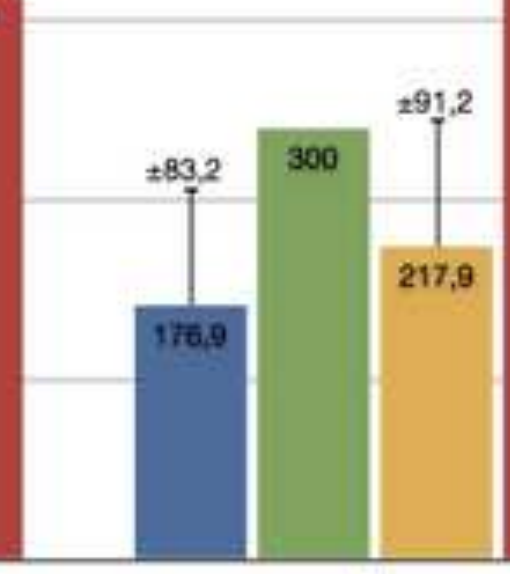

RH
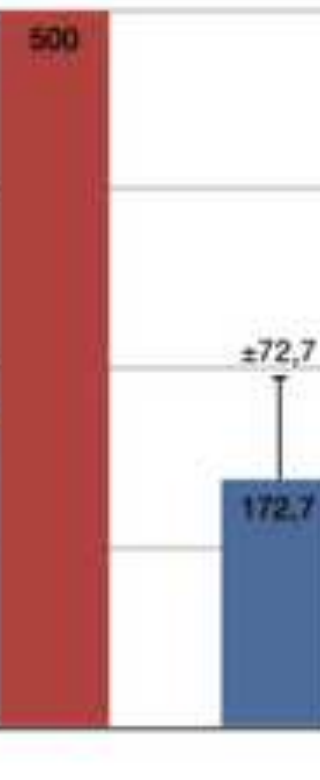

dose moyenne initialement prescrite dose maximale initialement prescrite dose moyenne atteinte à la dernière visite dose maximale atteinte à la dernière visite 